\title{
Cascade of field-induced magnetic transitions in a frustrated antiferromagnetic metal
}

\author{
A. I. Coldea, ${ }^{1,2,{ }^{*}}$ L. Seabra, ${ }^{3,4,2}$ A. McCollam, ${ }^{5}$ A. Carrington, ${ }^{2}$ L. Malone, ${ }^{2}$ A. F. Bangura,,${ }^{6,7,2}$ D. Vignolles, ${ }^{8}$ P. G.van Rhee, ${ }^{5}$ \\ R. D. McDonald, ${ }^{9}$ T. Sörgel, ${ }^{6}$ M. Jansen, ${ }^{6}$ N. Shannon, ${ }^{10,1,2}$ and R. Coldea ${ }^{1,2}$ \\ ${ }^{1}$ Clarendon Laboratory, Department of Physics, University of Oxford, Parks Road, Oxford OX1 3PU, United Kingdom \\ ${ }^{2}$ H. H. Wills Physics Laboratory, University of Bristol, Tyndall Avenue, Bristol BS8 1TL, United Kingdom \\ ${ }^{3}$ Department of Physics, Technion - Israel Institute of Technology, Haifa 32000, Israel \\ ${ }^{4}$ Max-Planck-Institut für Physik komplexer Systeme, 01187 Dresden, Germany \\ ${ }^{5}$ High Field Magnet Laboratory, IMM, Radboud University Nijmegen, 6525 ED Nijmegen, The Netherlands \\ ${ }^{6}$ Max-Planck-Institut fur Festkorperforschung, Heisenbergstrasse 1, 70569 Stuttgart, Germany \\ ${ }^{7}$ RIKEN (The Institute of Physical and Chemical Research), Wako, Saitama 351-0198, Japan \\ ${ }^{8}$ Laboratoire National des Champs Magnétiques Intenses (CNRS), 31077 Toulouse, France \\ ${ }^{9}$ National High Magnetic Field Laboratory, Los Alamos National Laboratory, MS E536, Los Alamos, New Mexico 87545, USA \\ ${ }^{10}$ Okinawa Institute of Science and Technology, 1919-1 Tancha, Onna-son, Kunigami, Okinawa 904-0495, Japan
}

(Received 23 December 2013; revised manuscript received 17 June 2014; published 7 July 2014)

\begin{abstract}
Frustrated magnets can exhibit many novel forms of order when exposed to high magnetic fields. Much less, however, is known about materials where frustration occurs in the presence of itinerant electrons. Here we report thermodynamic and transport measurements on micron-size single crystals of the triangular-lattice metallic antiferromagnet $2 \mathrm{H}-\mathrm{AgNiO}_{2}$, in magnetic fields of up to $90 \mathrm{~T}$ and temperatures down to $0.35 \mathrm{~K}$. We observe a cascade of magnetic phase transitions at 13.5, 20,28, and $39 \mathrm{~T}$ in fields applied along the easy axis, and we combine magnetic torque, specific heat, and transport data to construct the field-temperature phase diagram. The low-field experimental data are compared with theoretical calculations for a frustrated easy-axis Heisenberg model based on realistic parameters for the localized moments of $\mathrm{AgNiO}_{2}$. Deviations from this model's predictions are attributed to the role played by the itinerant electrons.
\end{abstract}

DOI: 10.1103/PhysRevB.90.020401

PACS number(s): 75.10.Jm, 67.90.+z, 71.18.+y, 71.27.+a

Frustrated magnets have proven to be a rich source of novel magnetic ground states such as spin liquids on triangular lattices and spin ices on pyrochlore lattices [1]. Most experimental and theoretical studies of frustrated magnetism have focused on insulating systems, but there are interesting examples of phenomena in metallic systems where frustration is believed to play a crucial role, such as heavy fermion physics in the spinel $\mathrm{LiV}_{2} \mathrm{O}_{4}$ [2] and the metallic spinliquid state in the pyrochlore $\mathrm{Pr}_{2} \mathrm{Ir}_{2} \mathrm{O}_{7}$ [3]. In this context, the layered delafossites $\mathrm{AgNiO}_{2}$ [4] and $\mathrm{Ag}_{2} \mathrm{NiO}_{2}$ [5] provide model systems for studying the interplay of metallic electrons and local-moment magnetism on a geometrically frustrated lattice.

Detailed structural studies on the hexagonal, $2 \mathrm{H}$-polytype $\mathrm{AgNiO}_{2}$ reveal a charge ordering transition at $365 \mathrm{~K}$, below which one-third of the $\mathrm{Ni}$ ions form a triangular lattice of localized $\mathrm{Ni}^{2+}(S=1)$ magnetic moments, while the remaining $\mathrm{Ni}^{3.5+}$ ions form a honeycomb network of itinerant paramagnetic sites [6-9]. The localized $\mathrm{Ni}^{2+}$ spins order magnetically below $T_{N}=19.5 \mathrm{~K}$ into a collinear antiferromagnetic (AFM) structure of alternating ferromagnetic stripes in the triangular plane, with spins aligned along the $c$ axis, while the itinerant $\mathrm{Ni}^{3.5+}$ sites remain paramagnetic [see Fig. 2(c)] [6,7]. Band structure calculations suggest that the Ag $s p$ band is entirely above the Fermi level and that the structure can be visualized as a magnetic insulator formed by $\mathrm{Ni}^{2+}$ (like $\mathrm{NiO}$ ) with a strong tendency to magnetic order, superimposed on a $\mathrm{Ni}^{3.5+}$ metal [6].

*Corresponding author: amalia.coldea@physics.ox.ac.uk
Here we report thermodynamic and transport measurements on micron-size single crystals of $2 \mathrm{H}-\mathrm{AgNiO}_{2}$ in magnetic fields of up to $90 \mathrm{~T}$ applied along the $c$ axis. We observe a cascade of magnetic transitions, and combine torque, heat capacity, and transport measurements to construct the fieldtemperature phase diagram. The results are compared with calculations for a frustrated easy-axis Heisenberg model for the localized $\mathrm{Ni}^{2+}$ moments, where a succession of field-driven phase transitions into canted antiferromagnetic phases is predicted in increasing applied field. The model captures several key experimental features at low and intermediate fields, in particular the anomalously strong temperature dependence of the phase boundary to the first canted phase, whereas the high-field phases are likely to be influenced by the itinerant electrons.

For this study, we used hexagonal-shaped single crystals (typical size $\sim 70 \times 70 \times 0.1 \mu \mathrm{m}^{3}$ ) grown under high oxygen pressure [4]. We performed a series of torque measurements (on more than 10 single crystals) using piezoresistive, selfsensing cantilevers, at low temperatures $(0.3 \mathrm{~K})$ both in static magnetic fields (up to $18 \mathrm{~T}$ in Oxford and Bristol, $33 \mathrm{~T}$ at the HFML in Nijmegen) and in pulsed fields (up to $55 \mathrm{~T}$ at the LNCMP, Toulouse and up to $90 \mathrm{~T}$ at NHMFL in Los Alamos). The longitudinal magnetization was measured by force magnetometry using a highly sensitive magnetometer developed in Nijmegen [10]. Specific heat was measured using a purpose built microcalorimeter using dc and relaxation techniques. The residual resistivity ratio is up to $\sim 250$, which indicates the high purity of the single crystals.

Magnetic torque in magnetic materials is caused by anisotropy, measuring the misalignment of the magnetization with respect to a uniform applied field. The torque exerted 

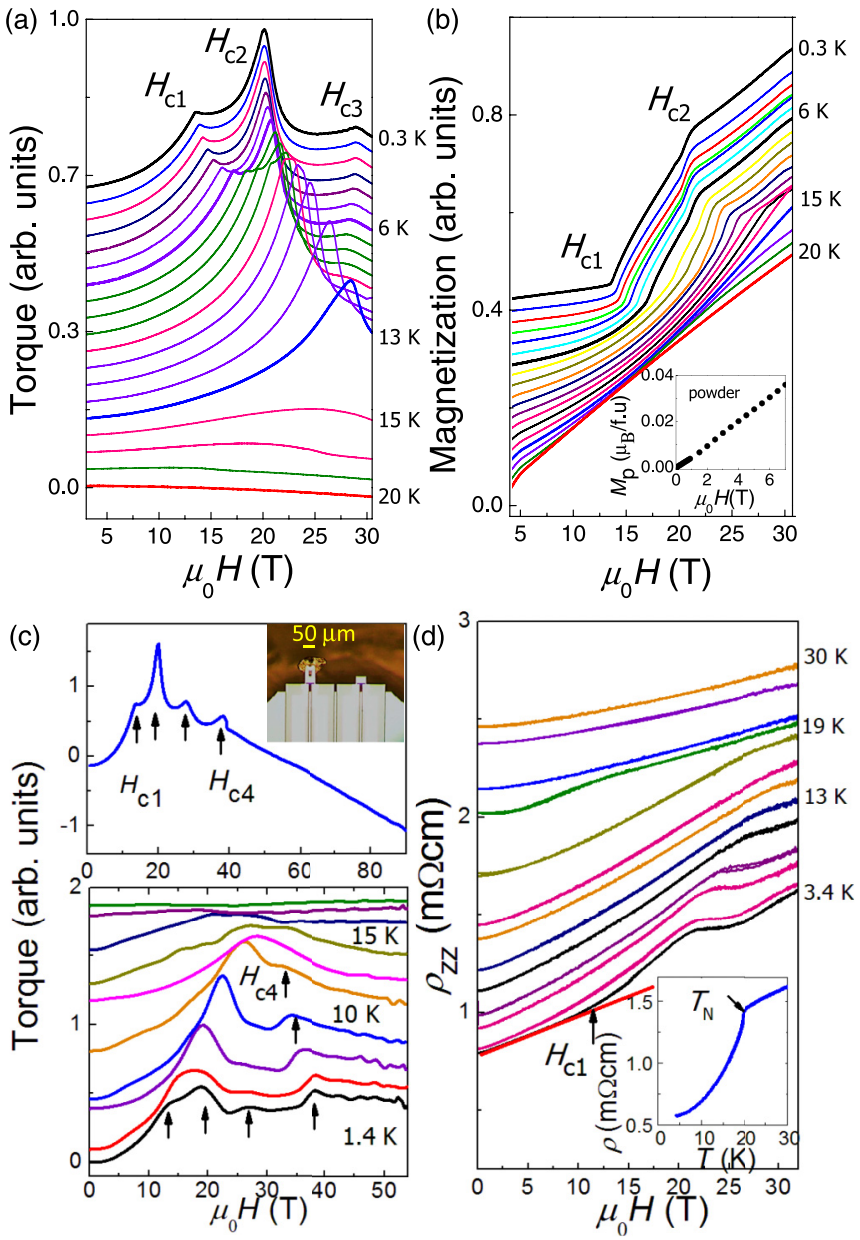

FIG. 1. (Color online) High magnetic field measurements of micron-size single crystals of $2 \mathrm{H}-\mathrm{AgNiO}_{2}$. Field dependence of (a) torque and (b) magnetization at constant temperature $T$ for field $\boldsymbol{H}$ nearly along the $c$ axis $\left(\theta \approx 3^{\circ}\right)$. The inset shows magnetization data for a powder sample at $5 \mathrm{~K}$. (c) Torque data up to $90 \mathrm{~T}$ measured at constant temperatures in pulsed magnetic fields on two different samples (top and bottom panels). Magnetic transitions are indicated by arrows and labels. Top inset shows a typical crystal mounted on a piezolever. (d) Field dependence of interlayer transport at constant temperatures below $30 \mathrm{~K}$ when $\boldsymbol{H} \| c$ (within $\theta \approx 3^{\circ}$ ). The arrow indicates the deviation from the linear dependence at $H_{c 1}$. The inset shows the low-temperature resistivity and the arrow indicates the position of the magnetic ordering transition at $T_{N}=19.5 \mathrm{~K}$. In all panels, traces at different temperatures are uniformly shifted vertically for clarity.

on a sample in an applied magnetic field $\boldsymbol{H}$ is $\boldsymbol{\tau}=\boldsymbol{M} \times \mu_{0} \boldsymbol{H}$, where $\boldsymbol{M}$ is the bulk magnetization. If $\boldsymbol{M}$ and $\boldsymbol{H}$ lie in the ac plane, then $\tau=\mu_{0}\left(M_{a} H_{c}-M_{c} H_{a}\right)=\frac{1}{2} \mu_{0}\left(\chi_{a}-\chi_{c}\right) H^{2}$ $\sin 2 \theta$, with $\theta=0$ when $\boldsymbol{H} \| c$. Thus, torque experiments measure the anisotropy of the magnetization in the $a c$ plane and the torque vanishes in field along the $c$ and $a$ axes (when $\sin 2 \theta=0$ ); the longitudinal magnetization provides access to the parallel $M_{c}$ component of the sample magnetization.

Figures 1(a) and 1(b) show the field and temperature dependence of the torque and magnetization, respectively, performed with the magnetic field aligned close to the easy axis $c$. At low temperatures and in low magnetic fields, the torque signal varies as $\tau \sim H^{2}$, implying a constant anisotropy, $\chi_{a}-\chi_{c}$, in the collinear AFM phase. By increasing the field, we observe kinks in torque at $\mu_{0} H_{c 1-4}=13.5,20,28$, and $39 \mathrm{~T}$ [see Figs. 1(a) and 1(c)], which we attribute to field-induced transitions. No further anomalies are detected at higher fields up to $90 \mathrm{~T}$ [see Fig. 1(c)]; however, the torque is finite and increases in absolute magnitude, indicating that the magnetization is not yet saturated and the region above $H_{c 4}$ is most likely a phase with spontaneous magnetic order. Further evidence for the phase transitions seen in torque data is provided by magnetization measurements shown in Fig. 1(b). At low fields, the magnetization has a weak, linear field dependence, and at $H_{c 1}$, the slope suddenly changes, suggesting a linear increase in the $M_{c}$ component in this phase, followed by a decrease in slope above $H_{c 2}$ and a small kink at $H_{c 3}$. The experiments of torque and specific heat in constant magnetic field as a function of temperature presented in Figs. 3(a) and 3(b) also clearly show the anomalies at $T_{N}$ and $T_{c 1}$. Later, we compare these measurements in detail with predictions for a spin Hamiltonian.

Another important fact about $2 \mathrm{H}-\mathrm{AgNiO}_{2}$ is that it is a good metal with low residual resistivity $(57 \mu \Omega \mathrm{cm})$ [see Fig. 1(d)] and quantum oscillations have been observed [11]. There is a significant contribution to the density of states at the Fermi level originating from the $\mathrm{Ni}$ sites on the honeycomb lattice [6]. Figure 1(d) shows that transport measurements also exhibit anomalies at the magnetic phase transitions, showing that the itinerant $d$ electrons are a sensitive probe of the magnetic ground state. There is a significant drop in resistivity below $T_{N}$ [see inset in Fig. 1(d)], which is likely the result of suppression of electronic scattering by low-energy spin fluctuations when a spin gap opens below $T_{N}$ [12]. Furthermore, magnetoresistance measurements in Fig. 1(d) indicate that in the vicinity of the magnetic transition, there is a clear change in slope at $H_{c 1}$ that fades away with increasing temperature. In zero field, the $\mathrm{Ni}^{2+}$ spins order in a collinear antiferromagnetic pattern, with spins pointing along the easy $c$ axis, schematically shown in Fig. 2(c) [6,12]. In magnetic fields applied along the easy axis, a transition is expected in a field of $\Delta /\left(g \mu_{B}\right)$ that matches the zero-field anisotropy spin gap, $\Delta$. Using the observed value of the first transition field $\mu_{0} H_{c 1}=13.5 \mathrm{~T}$ in Fig. 1(a) gives $\Delta=$ $1.57 \mathrm{meV}$ (using $g=2$ ), in good agreement with the value of $1.7(1) \mathrm{meV}$ estimated from inelastic neutron-scattering measurements [12]. For typical easy-axis antiferromagnets with unfrustrated interactions, the transition in field is to a spin-flop phase, where antiferromagnetically aligned spins are canted so that they have a net magnetization along the field. The spin-flop transition is signaled by an anomaly in torque [13-15].

The magnetic field-temperature phase diagram of $2 \mathrm{H}$ $\mathrm{AgNiO}_{2}$, based on magnetic torque, transport, and specificheat data obtained over a large range of fields and temperatures on different single crystals, is shown in Fig. 2(a). Unexpectedly, in this metallic magnet, we observe a cascade of phase transitions, suggesting the formation of different magnetic structures with increasing magnetic field (often referred to as metamagnetic transitions), with qualitative differences compared to conventional uniaxial antiferromagnets; see, e.g., Refs. [13,16-18]. In insulators, field-induced 

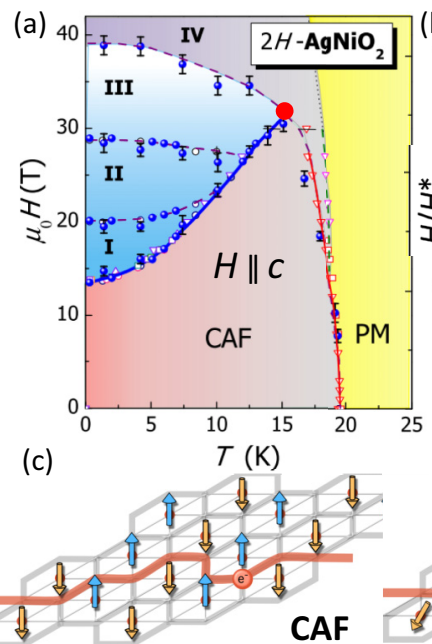

Collinear antiferromagnetic phase

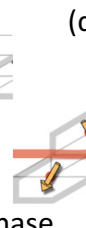

(d)
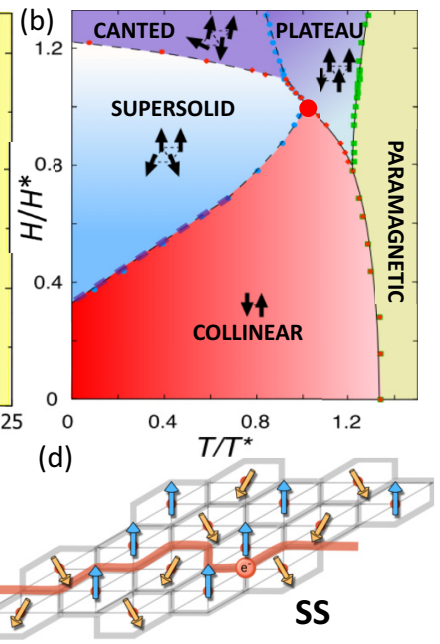

Supersolid phase

FIG. 2. (Color online) (a) The $H-T$ phase diagram of $2 H$ $\mathrm{AgNiO}_{2}$ from torque magnetometry (circles), specific heat (triangles), and transport (square). The solid and dashed lines indicate boundaries between different magnetic phases: collinear antiferromagnetic (AFM), field-induced phases I-IV, and paramagnetic (PM). (b) The phase diagram of the classical Heisenberg model on the triangular lattice with first-neighbor $\left(J_{1}=1\right)$ and second-neighbor $\left(J_{2}=0.15\right)$ in-plane interactions, coupling between layers $\left(J_{\perp}=-0.15\right)$, and easy-axis anisotropy $D=0.25$, obtained from Monte Carlo simulation [21]. The axes units are scaled to the point (large solid red circle) where the AFM, canted 1, and plateau phases meet with $T^{*}=0.3 J_{1}$, $B^{*}=1.6 J_{1}$. In the AFM phase, (c) the spin excitations are gapped, while in the canted 1 phase, (d) electrons can scatter from gapless spin excitations. The solid and dashed lines in the theoretical phase diagram indicate first-order and continuous transitions, respectively.

metamagnetic transitions were observed previously in twodimensional $\mathrm{CuFeO}_{2}(S=5 / 2)$, with a related delafossitetype structure [19].

To gain insight into the magnetism of $2 \mathrm{H}-\mathrm{AgNiO}_{2}$ in high magnetic fields, we consider below an effective spin model describing only the localized $S=1\left(\mathrm{Ni}^{2+}\right)$ spins interacting via a Heisenberg model including first- and second-neighbor antiferromagnetic exchange $\left(J_{1}, J_{2}\right)$ on the triangular lattice, a coupling between layers $\left(J_{\perp}\right)$, and easy-axis anisotropy $(D)$, using parameters obtained from fits to powder inelastic neutron-scattering data [12]. The resulting magnetic phase diagram in the easy-axis field obtained from classical Monte Carlo simulations was described in detail in Refs. [20,21] and in the Supplemental Material [22]. Here, we focus on the low-field region of the phase diagram of the spin model in Fig. 2(b), and we directly compare the measured and calculated thermodynamic quantities: torque, magnetization, and specific heat.

The experimental phase diagram in Fig. 2(a) shows that the phase boundary between AFM-phase I has an unusual fieldtemperature dependence, i.e., the transition field $H_{c 1}$ strongly increases upon increasing temperature. This behavior is well reproduced by the theoretical phase diagram in Fig. 2(b), where this phase boundary corresponds to a continuous transition from the collinear AFM into a four-sublattice "canted 1" phase, where two sublattices have spins "up" and the other two have spins "down" but canted away from the easy axis, as illustrated in Fig. 2(d). This structure has two coexisting spontaneous orders: the spin components along the field direction $(z)$ break the discrete translational symmetry of the lattice and the canted spin components of the down spins break the continuous spin rotational symmetry around the easy axis and pick a unique direction in the $x y$ plane. Because breaking of translational symmetry is analogous to ordering in a crystal (solid) and breaking of rotational symmetry is analogous to a transition to a superfluid, the canted 1 phase has been called a magnetic analog of a supersolid $[20,21,23,24]$. The canted 1 phase arises continuously from the collinear AFM phase by tilting the down spins, as opposed to a spin-flop phase where the transition from the AFM phase is first order with all spins flopping nearly perpendicular to the applied field.

Next, we directly compare the experimental results for magnetic torque, magnetization, and specific heat with those predicted by the model described above. The temperature dependence of the torque at fixed field has a very similar shape and qualitative form in the experiment and theoretical model [see Figs. 3(a) and 3(b)]: in both cases, upon cooling from high temperatures in the paramagnetic phase, the torque changes sign below $T_{N}$, increases upon decreasing temperature in the AFM phase, then has a peak at $T_{c 1}$, identified with the transition into the canted 1 phase. The temperature dependence of the specific-heat data in constant magnetic fields also shows consistent behavior between experiment and theory; see Figs. 3(c) and 3(d). At low fields, a single anomaly is observed at the $T_{N}$ transition. In fields above $H_{c 1}$, a second anomaly is observed at low temperatures $T_{c 1}$ identified with the transition AFM-canted 1, and this shifts to higher temperatures upon increasing field [25]. At higher fields, an additional anomaly (labeled as $T^{\prime}$ ) appears near $T_{N}$. In the theoretical model, this double feature bounds an intermediate collinear phase, labeled plateau in Fig. 2(b), with a four-spin unit cell, which has a $T=0 \mathrm{~K}$ magnetization of half of the saturated moment, which is remarkable for a triangular-lattice system.

Following the same approach, we compare the measured field dependencies of magnetic torque and magnetization at constant temperature, shown in Figs. 1(a) and 1(b), with those predicted by the model in Figs. 3(e) and 3(f). At very low temperatures in the AFM phase, the measured and simulated magnetization are both linear in $H$, whereas the torque shows a quadratic dependence, $H^{2}$, as a function of magnetic field, and displays a kink at the first critical field, $H_{c 1}$, which becomes sharper upon lowering temperature. Furthermore, inside the canted 1 phase at fields right above the transition $H_{c 1}$, magnetic torque is observed to be almost independent of the applied field [see Fig. 3(e)]. All of these observations are at odds with standard spin-flop transitions (see Refs. [13-15,17,18]); in those cases, torque should show a strong divergence at a spin-flop transition field $H_{c}$, then be strongly suppressed above $H_{c}$, whereas the magnetization would have an abrupt jump at $H_{c}$, indicative of the first-order nature of this transition. The transition field for a spin-flop transition is usually independent of temperature, since it is determined by a balance of energies between different spin configurations rather than entropy.

While the classical spin model for the localized $\mathrm{Ni}^{2+}$ moments can provide a good description of many of the intriguing qualitative and quantitative features of the lowest 

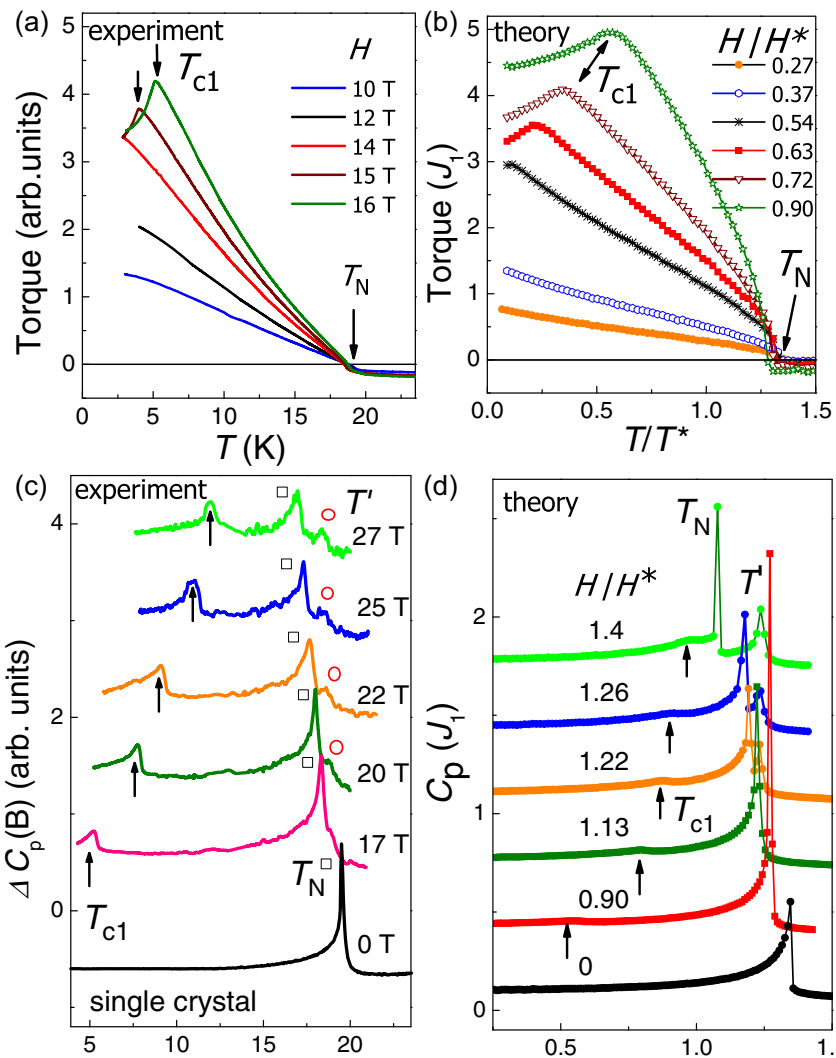

(d)
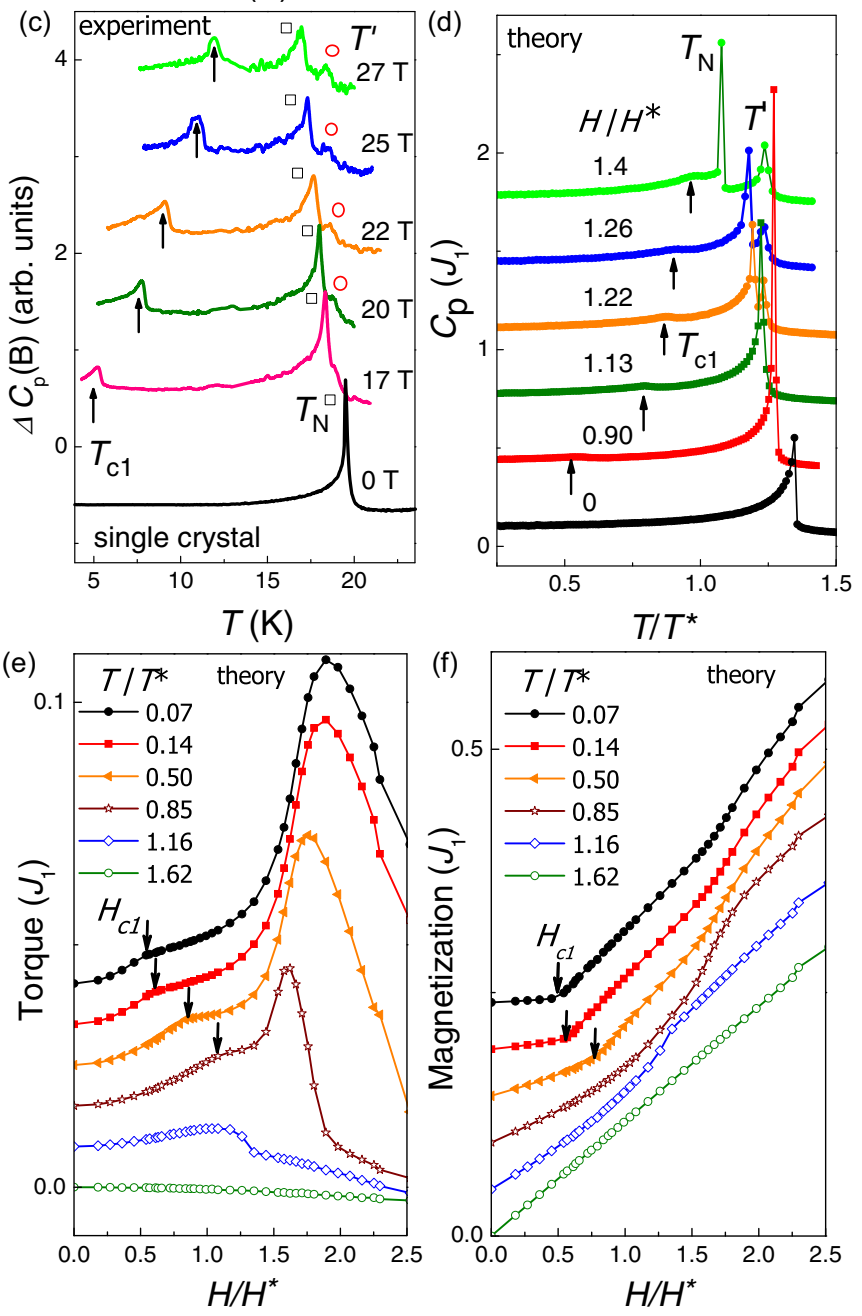

FIG. 3. (Color online) Temperature dependence of torque at fixed applied field in (a) experiments and (b) theoretical model. Temperature dependence of specific heat at fixed field in (c) experiment and (d) theory. Identified transitions are indicated by arrows at $T_{c 1}$, squares at $T_{N}$, and circles at $T^{\prime}$. Calculated (e) torque and (f) magnetization as a function of applied field at constant temperatures compared with experimental data in Figs. 1(a) and 1(b) $\left(\theta=5^{\circ}\right)$. In the theoretical model, saturation occurs for $H / H^{*} \approx 4.8$, with $T^{*}$ and $H^{*}$ defined in Fig. 2(b). Traces at (c),(d) different fields and (e),(f) different temperatures are shifted vertically for clarity.

field-induced transition observed at $H_{c 1}$, the model cannot capture the full phase diagram and, in particular, cannot account for the presence of multiple phases spanning relatively narrow field ranges above $H_{c 1}$ (labeled as I-III). It may be possible that itinerant electrons, neglected in the spin model, could affect the phase diagram in this region of intermediate fields and potentially lead to additional transitions at $H_{c 2}$ and $H_{c 3}$ inside the canted 1 phase of the classical model. In the canted 1 phase, the broken translational symmetry may lead to reconstruction of the Fermi surface, while the particular points in the Brillouin zone that may have gapless Goldstone modes may lead to the inelastic scattering of electrons and thus an increase of resistivity above $H_{c 1}$, as observed in Fig. 1(d). A possible reconstruction of the Fermi surface at the low-field transition may also explain why more entropy is released in experiment than in theory at the AFM to canted 1 transition, indicated by the large anomaly in specific heat at $T_{c 1}$ in Figs. 3(c) and 3(d). Within the localized model, quantum fluctuations or the ratios of exchange interaction strengths may alter the precise shape of the boundaries between different phases, but are not expected to change the overall behavior of the phase diagram.

The observed $\mathrm{H}-\mathrm{T}$ phase diagram of $2 \mathrm{H}-\mathrm{AgNiO}_{2}$ reflects the complexity of its magnetic interactions. Since localized magnetic moments are embedded in a metal, they are subject to Ruderman-Kittel-Kasuya-Yosida (RKKY) interactions, which, unlike superexchange, decay slowly with distance and may provide non-negligible further-neighbor exchange. Furthermore, the band structure calculations show a small but finite magnetic moment $\left(m_{i} \approx 0.1-0.2 \mu_{B}\right)$ [6] on the itinerant and inherently nonmagnetic Ni sites on the honeycomb [see Fig. 2(c)]. The Hund's rule coupling on these sites may provide an additional incentive for the itinerant electron spins to order magnetically as proposed for the metallic ferromagnet in $\mathrm{SrRuO}_{3}$ [26].

In conclusion, we have probed the magnetic phase diagram of the frustrated antiferromagnetic metal $2 \mathrm{H}-\mathrm{AgNiO}_{2}$ in strong magnetic fields applied along the easy axis and have observed a cascade of magnetic phase transitions. Thermodynamic measurements have been compared with predictions of an effective localized spin model, which explains key features of the phase diagram and proposes that the first field-induced transition is to a canted antiferromagnetic phase with two broken symmetries, analogous to a magnetic supersolid. To confirm such an exotic magnetic state, direct measurements of the magnetic structure would be required using resonant $\mathrm{x}$-ray or neutron diffraction. However, a more realistic model for $2 \mathrm{H}-\mathrm{AgNiO}_{2}$ needs to also consider the itinerant $d$ electrons on the honeycomb lattice, which may participate in the exchange interactions and may affect the magnetic order of the localized moments. Therefore, the itinerant electrons may be responsible for some of the higher-field transitions observed both in transport and thermodynamic measurements. Further studies will explore how those phase transitions correlate with changes of the Fermi-surface topology in this frustrated magnetic metal, where $d$ electrons have mixed localized and itinerant character.

We thank J. Analytis, C. Jaudet, P. A. Goddard, Jos Perenboom, and M. D. Watson for technical support during experiments. We thank I. I. Mazin, A. Schofield, and I. Vekhter for useful discussions. This work was supported by EPSRC Grants No. EP/I004475/1, No. EP/C539974/1, No. EP/G031460/1, and No. EP/I017836/1, FCT Grant No. 
SFRH/BD/27862/2006, and for part of the work we acknowledge the support of HFML-RU/FOM and LNCMICNRS, a member of the European Magnetic Field Laboratory
(EMFL). A.I.C. acknowledges support from an EPSRC Career Acceleration Fellowship (Grant No. EP/I004475/1). R.D.M. acknowledges support from BES "Science at 100 T."
[1] For a review, see L. Balents, Nature (London) 464, 199 (2010).

[2] C. Urano, M. Nohara, S. Kondo, F. Sakai, H. Takagi, T. Shiraki, and T. Okubo, Phys. Rev. Lett. 85, 1052 (2000).

[3] S. Nakatsuji, Y. Machida, Y. Maeno, T. Tayama, T. Sakakibara, J. van Duijn, L. Balicas, J. N. Millican, R. T. Macaluso, and J. Y. Chan, Phys. Rev. Lett. 96, 087204 (2006).

[4] T. Sörgel and M. Jansen, Z. Anorg. Allg. Chem. 631, 2970 (2005).

[5] H. Yoshida, Y. Muraoka, T. Sörgel, M. Jansen, and Z. Hiroi, Phys. Rev. B 73, 020408 (2006).

[6] E. Wawrzynska, R. Coldea, E. M. Wheeler, I. I. Mazin, M. D. Johannes, T. Sörgel, M. Jansen, R. M. Ibberson, and P. G. Radaelli, Phys. Rev. Lett. 99, 157204 (2007).

[7] E. Wawrzynska, R. Coldea, E. M. Wheeler, T. Sörgel, M. Jansen, R. M. Ibberson, P. G. Radaelli, and M. M. Koza, Phys. Rev. B 77, 094439 (2008).

[8] I. I. Mazin, D. I. Khomskii, R. Lengsdorf, J. A. Alonso, W. G. Marshall, R. M. Ibberson, A. Podlesnyak, M. J. Martínez-Lope, and M. M. Abd-Elmeguid, Phys. Rev. Lett. 98, 176406 (2007).

[9] G. L. Pascut, R. Coldea, P. G. Radaelli, A. Bombardi, G. Beutier, I. I. Mazin, M. D. Johannes, and M. Jansen, Phys. Rev. Lett. 106, 157206 (2011).

[10] A. McCollam, P. G. van Rhee, J. Rook, E. Kampert, U. Zeitler, and J. C. Maan, Rev. Sci. Instrum. 82, 053909 (2011).

[11] A. I. Coldea et al., arXiv:0908.4169.

[12] E. M. Wheeler, R. Coldea, E. Wawrzynska, T. Sörgel, M. Jansen, M. M. Koza, J. Taylor, P. Adroguer, and N. Shannon, Phys. Rev. B 79, 104421 (2009).

[13] H. Uozaki, T. Sasaki, S. Endo, and N. Toyota, J. Phys. Soc. Jpn. 69, 2759 (2000).

[14] T. Nagamiya, K. Yosida, and R. Kubo, Adv. Phys. 4, 1 (1955).

[15] A. N. Bogdanov, A. V. Zhuravlev, and U. K. Rößler, Phys. Rev. B 75, 094425 (2007).
[16] T. Kawamoto, Y. Bando, T. Mori, T. Konoike, Y. Takahide, T. Terashima, S. Uji, K. Takimiya, and T. Otsubo, Phys. Rev. B 77, 224506 (2008)

[17] C. C. Becerra, N. F. Oliveira, A. Paduan-Filho, W. Figueiredo, and M. V. P. Souza, Phys. Rev. B 38, 6887 (1988).

[18] R. Toft-Petersen, N. H. Andersen, H. Li, J. Li, W. Tian, S. L. Bud'ko, T. B. S. Jensen, C. Niedermayer, M. Laver, O. Zaharko, J. W. Lynn, and D. Vaknin, Phys. Rev. B 85, 224415 (2012).

[19] N. Terada, Y. Narumi, Y. Sawai, K. Katsumata, U. Staub, Y. Tanaka, A. Kikkawa, T. Fukui, K. Kindo, T. Yamamoto, R. Kanmuri, M. Hagiwara, H. Toyokawa, T. Ishikawa, and H. Kitamura, Phys. Rev. B 75, 224411 (2007).

[20] L. Seabra and N. Shannon, Phys. Rev. Lett. 104, 237205 (2010).

[21] L. Seabra and N. Shannon, Phys. Rev. B 83, 134412 (2011).

[22] See Supplemental Material at http://link.aps.org/supplemental/ 10.1103/PhysRevB.90.020401 for details on the effective model and its simulation with classical Monte Carlo.

[23] H. Matsuda and T. Tsuneto, Prog. Theor. Phys. Suppl. 46, 411 (1970).

[24] K. S. Liu and M. E. Fisher, J. Low Temp. Phys. 10, 655 (1973).

[25] In the limit of our experimental resolution (specific-heat experiments are performed on extremely small samples) as a function of temperature (experiments were made on $70 \times 70 \times 10 \mu \mathrm{m}^{3}$ crystals), we cannot detect clear signatures for the other two transitions clearly observed both in the torque and transport measurements. This could be due to the fact that the scans were performed mainly at constant temperature and transition boundaries are nearly flat and/or that these transitions are related to a reorientation of spins which does not imply a significant change in entropy and do not result in a large anomaly in the specific heat.

[26] I. I. Mazin and D. J. Singh, Phys. Rev. B 56, 2556 (1997). 